\title{
IGF-1 DEFICIENCY IN NEWLY DIAGNOSED GRAVES’ DISEASE PATIENTS
}

\section{Sorina Martin ${ }^{1,2}$, Minodora Betivoiu', Suzana Florea ${ }^{3}$, Simona Fica ${ }^{1,2}$}

1. Elias Hospital, Endocrinology Department

2. Carol Davila University of Medicine and Pharmacy, Endocrinology Department

3. Elias Hospital, Laboratory Department

\section{OBJECTIVES}

Thyroid hormones influence GH/IGF-1 axis, but previous studies reported discrepant results regarding serum

IGF-1 levels in hyperthyroidism. We have therefore investigated, at diagnosis, the relationship between serum IGF-1 levels/IGF-1 z scores and clinical and biological characteristics of Graves' disease (GD) patients. We also compared IGF-1 levels/IGF-1 z scores, at diagnosis, between GD and autonomous hyperthyroidism patients.

\section{METHODS}

This cross-sectional study included 119 newly diagnosed hyperthyroid patients (98 with GD, 21 with toxic multinodular goiter) that presented consecutively to our clinic.

The main measured parameters: TSH, FT4, FT3, TT3, thyroglobulin, TPOAb, ATA, TRAb, IGF-1. Patients were considered IGF-deficient if IGF-1 z score was $\leq-2 S D$ from mean for age.

\section{RESULTS}

In GD patients men had higher IGF-1 levels $(p=0.023)$ and IGF-1 z scores $(p=0.013)$ than women. $18.4 \%$ of $G D$ patients were, at diagnosis, IGF-1 deficient. Compared to patients without IGF-1 deficiency, these patients presented, at diagnosis, higher thyroglobulin (median=72.55, IQR=116.02 vs median=11.40, IQR=80.74ng/ml, $p=0.002$ ), $F T 3$ (median=11.30, IQR=7.64 vs median=7.33, IQR=5.72pg/ml, $\mathrm{p}=0.027$ ) and lower ATA (median=20, IQR=0 vs median=34.05, IQR=161UI/ml, $\mathrm{p}=0.001$ ) levels. Thyroglobulin was identified as strong predictor for IGF-1 deficiency (AUROC=0.732, 95\%IC:0.620-0.844, $\mathrm{p}=0.002$; cut-off for thyroglobulin=50.40ng/ml, Se=77.8\%, Sp=70\%).

IGF-1 status wasn't influenced by gender $(p=0.08)$, current smoking $(p=0.55)$, goiter size $(p=0.53)$, ophtalmopathy $(p=0.33)$, TRAb $(p=0.23)$, TPOAb status $(p=0.36)$.

The prevalence of IGF-1 deficiency was higher in GD patients compared to patients with toxic goiter $(18 \%$ vs $0 \%$, $\left.x^{2}=4.54, p=0.033\right)$

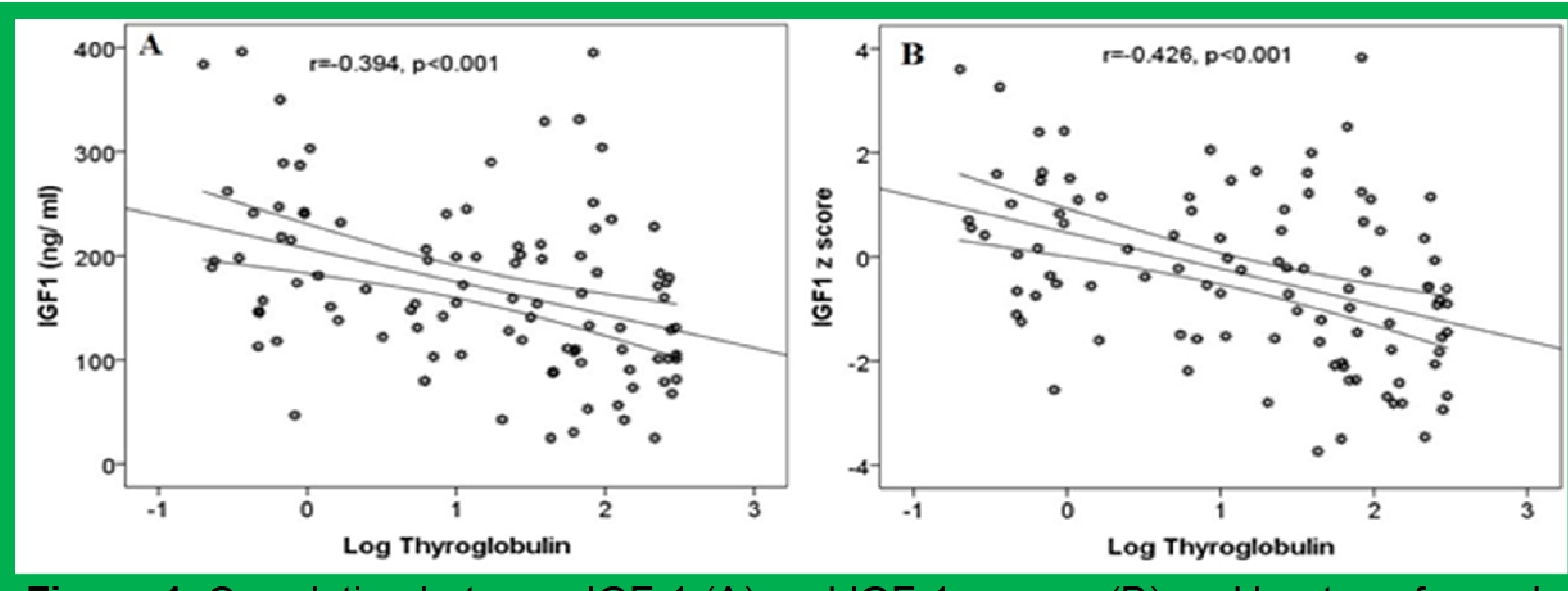

Figure 1. Correlation betwe
serum thyroglobulin levels

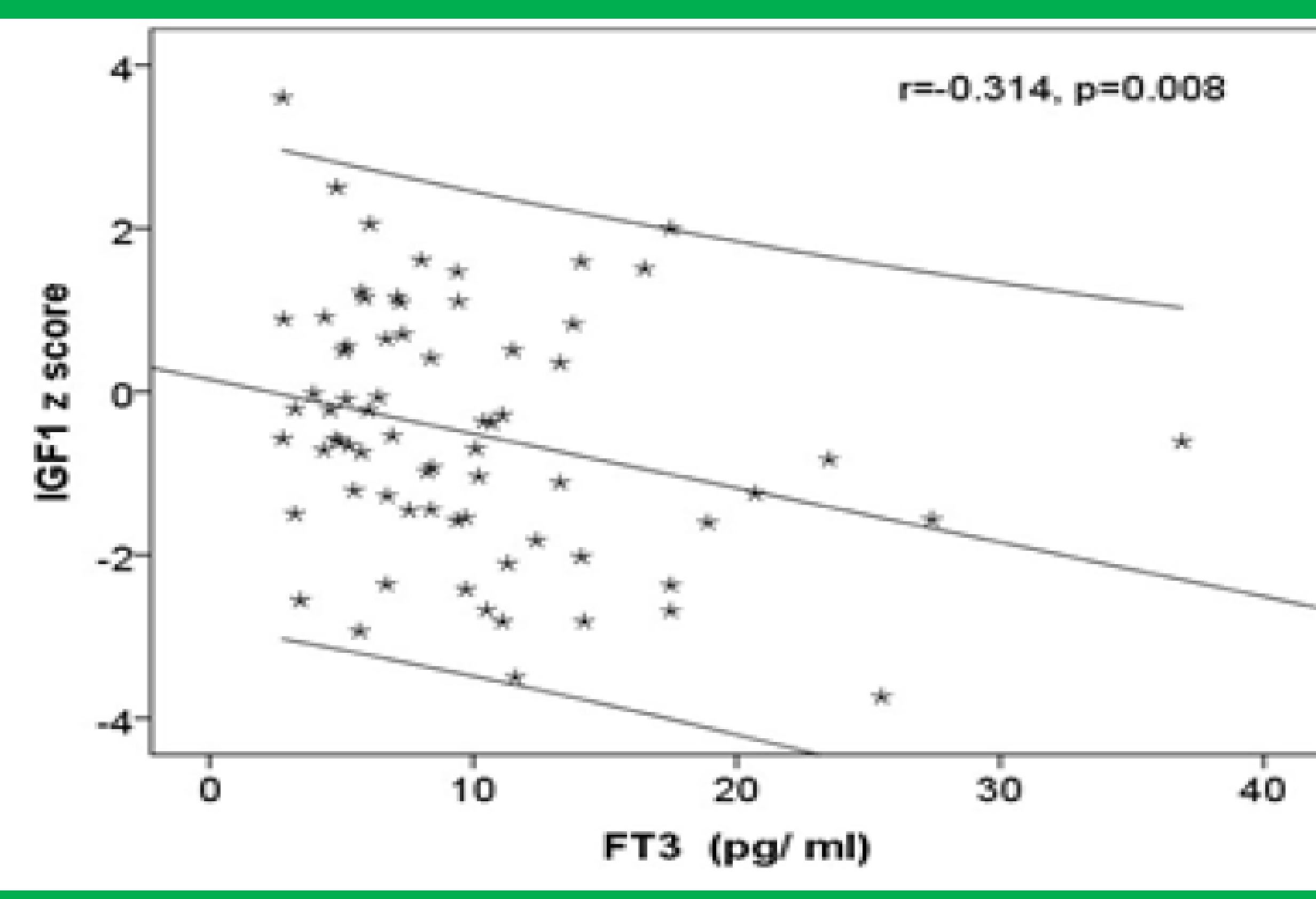

Figure 3. Correlation between IGF-1 z score and FT3 at diagnosis
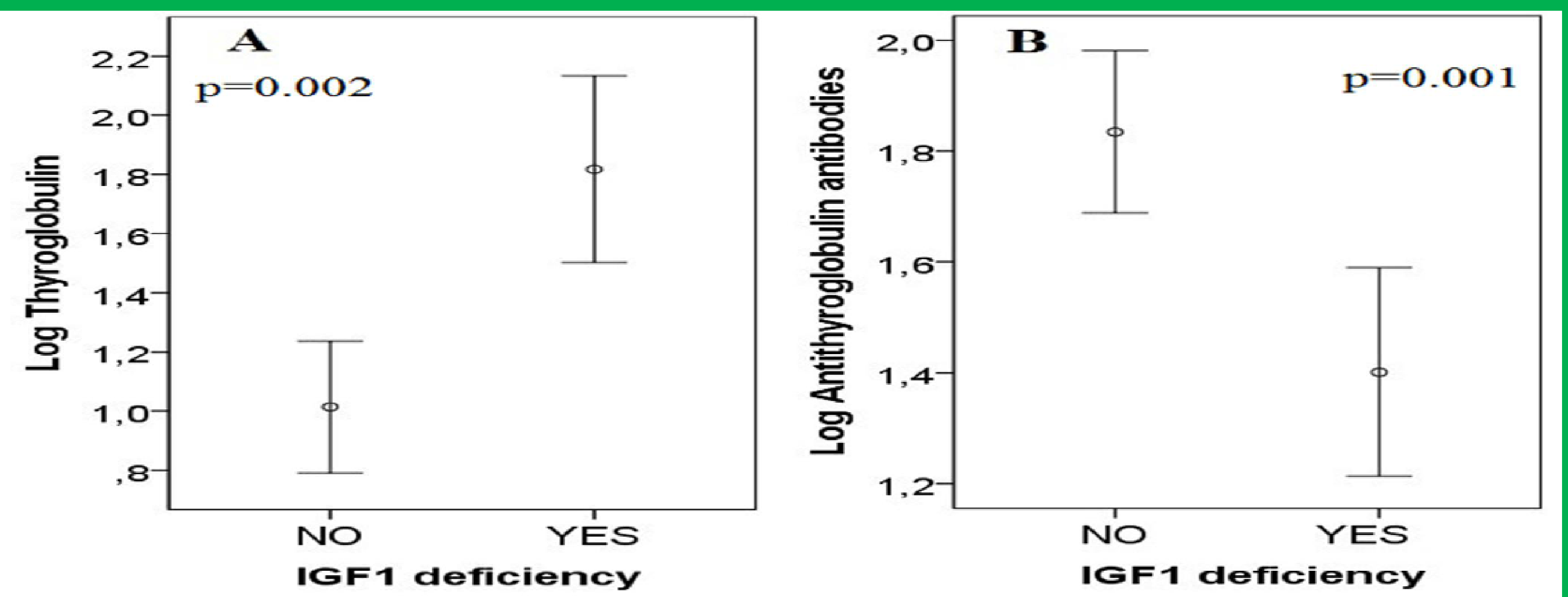

Figure 2. Mean log transformed antithyroglobulin antibodies (A) and

thyroglobulin (B) values, at diagnosis, according to IGF-1 status

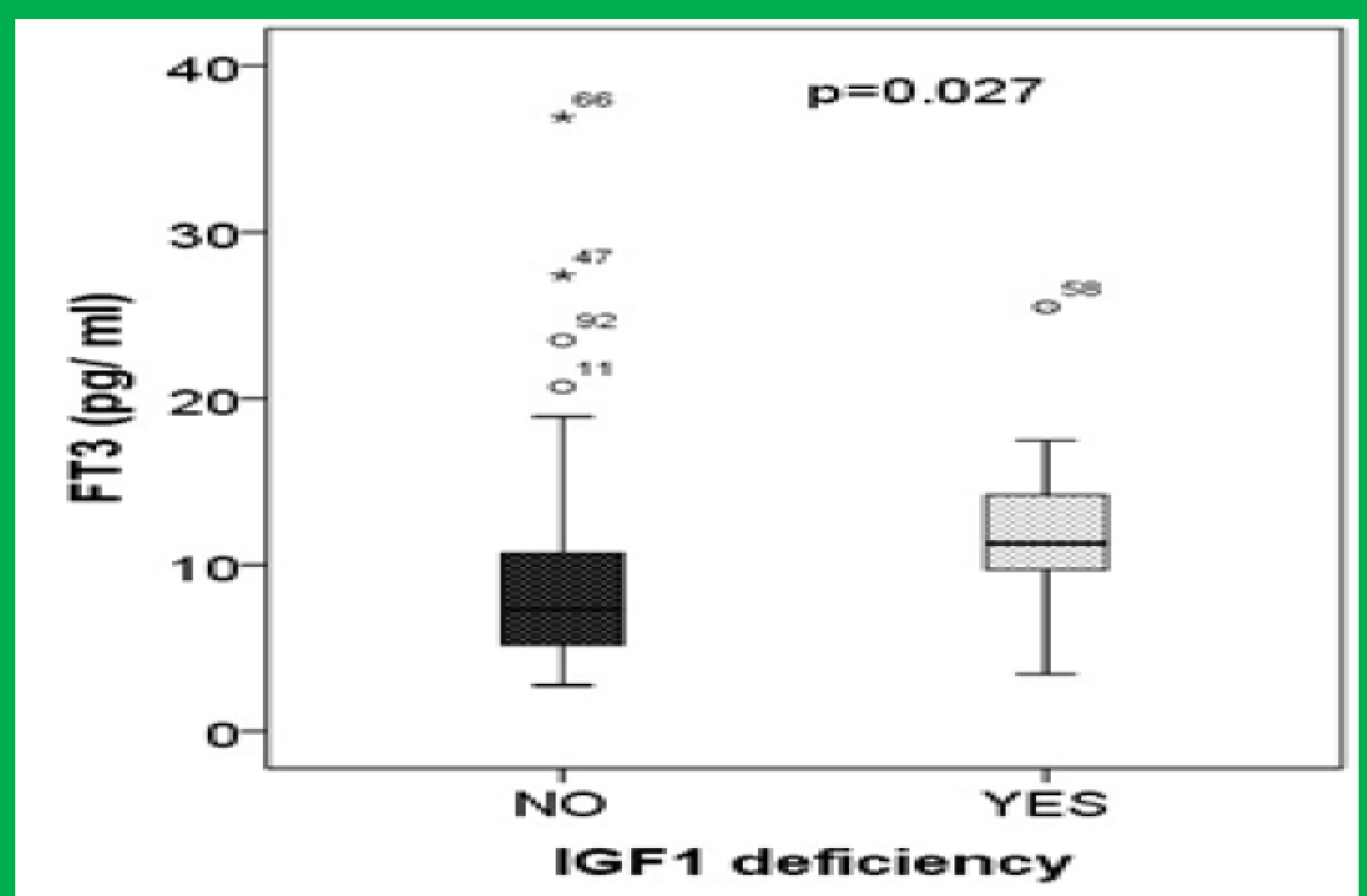

Figure 4. Serum FT3 levels according to IGF-I status, at diagnosis

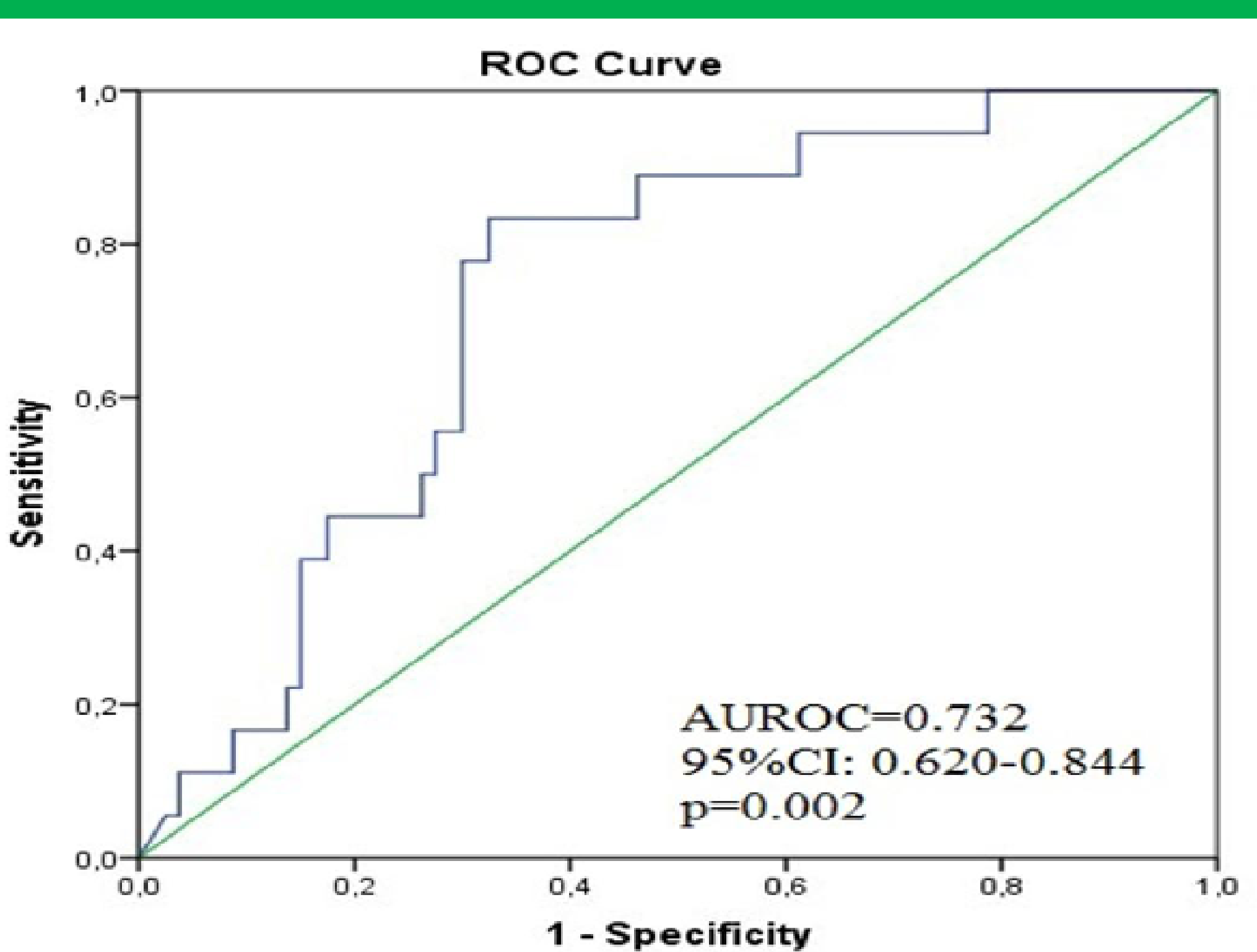

Figure 5. Predictive value of serum thyroglobulin levels on IGF-1 deficiency
CONCLUSIONS

Our study shows, for the first time, the presence of IGF-1 deficiency in nearly one fifth of newly diagnosed GD patients. IGF-1 deficiency was associated with lower ATA titers, higher thyroglobulin levels and more severe FT3 hyperthyroidism.

The presence of active GO didn't influence IGF-1 status.GD patients had higher prevalence of IGF-1 deficiency than patients with toxic multinodular goiter

\section{References}

Glynn N et al, 2012 Diagnosing growth hormone deficiency in adults. Int J Endocrinol 2012:972617

Lakatos $\mathrm{P}$ et al, 2000 Serum insulin-like growth factor-I, insulin-like growth factor binding proteins, and bone mineral content in hyperthyroidism. Thyroid 10:417-423

Zimmermann-Belsing T et al, 2004 The insulin-like growth axis in patients with autoimmune thyrotoxicosis: effect of antithyroid drug treatment. Growth Horm IGF Res 14:235-244 